\title{
Nonlinear Finite Element Solution of Post-buckling Responses of FGM Panel Structure under Elevated Thermal Load and TD and TID Properties
}

\author{
Subrata Kumar Panda ${ }^{1, a}$, Trupti Ranjan Mahapatra ${ }^{2}$ and Vishesh Ranjan $\mathrm{Kar}^{3}$ \\ ${ }^{1}$ NIT Rourkela, 769008, Odisha, India \\ ${ }^{2}$ KIIT University, Bhubaneswar, 751024, Odisha, India \\ ${ }^{2}$ VIT University, Vellore, 632014, Tamil Nadu, India
}

\begin{abstract}
The nonlinear finite element solutions for the buckling and post-buckling responses of the functionally graded shell panel subjected to the non-uniform thermal environment have been presented in this article. The thermal fields are assumed as uniform, linear and nonlinear temperature rise across the thickness of shell panel and the properties of each constituent are considered to be temperature dependent. The effective material properties of the graded structure are evaluated using the Voigt's micromechanical rule in conjunction with power-law distribution. For the analysis purpose, a general nonlinear mathematical model of the functionally graded shell panel has been developed based on the higher order shear deformation theory and Green-Lagrange type geometrical nonlinear strains. The system governing equation of the panel structure is derived using the variational principle. Further, suitable nonlinear finite element steps have been adopted to discretize the model for the computation of the desired responses in association with the direct iterative method. The convergence and the validation behavior of the present numerical model are initially tested to demonstrate its efficacy and significance. Finally, the effects of curvature, power law index and different support conditions on the buckling and post-buckling responses of the functionally graded shell panels are investigated and discussed in details.
\end{abstract}

\section{Introduction}

The ability of the functionally graded materials (FGMs) to withstand very high thermal gradient have made them popular in many high performance engineering applications under extreme temperature loading conditions. During service under sever thermal load, the FGM structures buckles and geometrical nonlinearity is induced. The consideration of the temperature dependent (TD) material properties (rather merely considering temperature independent (TID) material properties alone) is very much essential for the exact analysis of their stability behaviour. The linear and nonlinear buckling and post-buckling behaviour of FGM flat/curved panel subjected to uniform and non-uniform temperature loading conditions was investigated numerically by various researchers in past by using the existing/refined shear deformation theories such as the Classical Plate Theory (CPT) [1-2], First

\footnotetext{
${ }^{a}$ Corresponding author: call2subrat@gmail.com, pandask@nitrkl.ac.in
} 
Order Shear Deformation Theory (FOSDT) [3] and High Order Shear Deformation Theory (HOSDT) [5]. It is evident from the review of literature that, the FEM based numerical studies related to the geometrically nonlinear buckling and the post-buckling behavior of FG spherical panels is very limited [6-7]. Also, studies using the CPT [8-9] /FOSDT [10-12] mid-plane kinematics are more in number in comparison to the HOSDT [13-15]. Researchers considered the through the thickness temperature variation to be uniform, linear and nonlinear and incorporated TD and TID material properties of the FG structures. However, in all the aforementioned research to represent the geometrical distortion the von-Karman type of nonlinear kinematics has been adopted in contrast to the Green-Lagrange type that accounts for the deformation behaviour of material continuum more accurately [16].

The motivation of the present research is to study the buckling and the post-bucking behavior of FG spherical shell panel by considering Green-Lagrange type geometrical nonlinearity and the HOSDT mid-plane kinematics. Moreover, the FG shell panel properties are evaluated using Voigt's rule of mixture in conjunction with the power-law of distribution by taking both the TD and TID material properties. The governing equation of FG panel is derived using the variational principle and discretised using a nine noded isoparametric Lagrangian element with nine degrees of freedom per node. A direct iterative method is employed to obtain the desired responses.

\section{Nonlinear finite element formulations}

Figure 1 shows a typical FG spherical shell panel with a rectangular base with length ' $a$ ', width ' $b$ ' and uniform thickness ' $h$ ', considered for the present analysis. The curvature at the mid surface (at $z=$ 0 ) of the shell panel is defined as ' $R$ '. The displacement field based on the HOSDT mid-plane kinematics as in [17]:

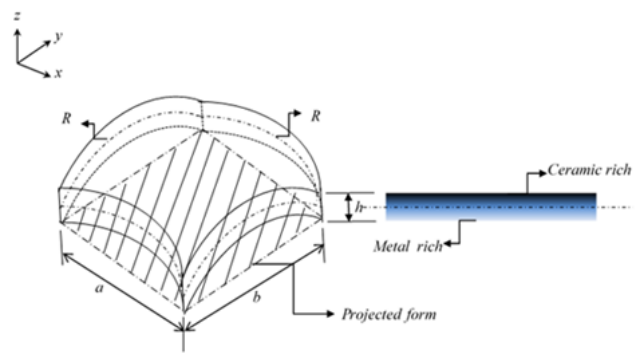

Figure 1. Geometry and dimension of FG spherical shell panel.

$$
\begin{aligned}
& u(x, y, z, t)=u_{0}(x, y, t)+z \theta_{x}(x, y, t)+z^{2} u_{0}^{*}(x, y, t)+z^{3} \theta_{x}^{*}(x, y, t) \\
& v(x, y, z, t)=v_{0}(x, y, t)+z \theta_{y}(x, y, t)+z^{2} v_{0}^{*}(x, y, t)+z^{3} \theta_{y}^{*}(x, y, t) \\
& w(x, y, z, t)=w_{0}(x, y, t)
\end{aligned}
$$

where, $\left(u_{0}, v_{0}, w_{0}\right)$ represent the corresponding displacements of the points on the mid-plane along $(x, y, z)$ coordinates, respectively. $\theta_{y}$ and $\theta_{x}$ are the rotations of normal to the mid-plane about the $x$ and $y$ axis, respectively and $\left(u_{0}^{*}, v_{0}^{*}, \theta_{x}^{*}, \theta_{y}^{*}\right)$ are the higher order terms of the Taylor series expansion defined at the mid-plane those account for the parabolic distribution of shear stress.

The small strain and large deformation behavior of the FG shell panel is expressed by considering Green-Lagrange type nonlinear strain-displacement field as in [18]

$$
\{\varepsilon\}=\left\{\varepsilon_{l}\right\}+\left\{\varepsilon_{n l}\right\}
$$

where, $\left\{\varepsilon_{l}\right\}$ and $\left\{\varepsilon_{n l}\right\}$ are the linear and the nonlinear strain tensors, respectively. 
The temperature dependent material properties of the graded structure have been obtained using the equivalent steps as in the reference [19]. Now, the effective elastic and the thermal properties of the FGM are obtained via Voigt's micromechanics model using the following equation [15]:

$$
P(T, z)=\left\{P_{c}(T, z)-P_{m}(T, z)\right\}\left(\frac{z}{h}+\frac{1}{2}\right)^{n}+P_{m}(T, z)
$$

where, subscript ' $m$ ' and ' $c$ ' represent the ceramic and the metal constituents, respectively and $P_{0}, P_{-1}, P_{1}, P_{2}$ and $P_{3}$ are the coefficients of temperature $(T)$ and $n(0 \leq n<\infty)$ is the powerlaw index.

The temperature function is expressed in the following form for uniform temperature variation:

$$
T=T_{m}+\Delta T \text { or } T=T_{c}
$$

where, $\Delta T=T_{c}-T_{m}$ and $T_{m}$ and $T_{c}$ are the temperatures at the bottom and top surfaces of the panel, respectively. The detail of the linear temperature field through the thickness condition the temperature field is expressed as can be seen in Kar and Panda [16].

The thermo-elastic stress tensor at any point within the FG spherical shell panel can be written as

$$
\{\sigma\}=\left\{\begin{array}{lllll}
\sigma_{x x} & \sigma_{y y} & \sigma_{x y} & \sigma_{x z} & \sigma_{y z}
\end{array}\right\}^{T}=[C]\left(\{\varepsilon\}-\left\{\varepsilon_{t h}\right\}\right)
$$

where, $[Q]$ is the reduced stiffness matrix and $\left\{\varepsilon_{t h}\right\}=\left\{\begin{array}{lllll}1 & 1 & 0 & 0 & 0\end{array}\right\}^{T} \alpha(T, z) \Delta T$ is the thermal strain tensor at any point within the shell panel and the details of [C] can be seen in [16].

The elemental displacement vector is expressed using the corresponding shape function and the nodal displacement field as

$$
\left\{\lambda_{0}\right\}=\sum_{i=1}^{9} N_{i}\left\{\lambda_{0_{i}}\right\}
$$

where, $\left\{\lambda_{0_{i}}\right\}=\left[\begin{array}{lllllllll}u_{0_{i}} & v_{0_{i}} & w_{0_{i}} & \theta_{x_{i}} & \theta_{y_{i}} & u_{0_{i}}^{*} & v_{0_{i}}^{*} & \theta_{x_{i}}^{*} & \theta_{y_{i}}^{*}\end{array}\right]^{T}$ is the nodal displacement vector and $N_{i}$ is the shape function for the $i^{\text {th }}$ node, respectively. The details of the shape function can be seen in [19].

The linear and nonlinear mid-plane strain vector in terms of nodal displacement vector is written as

$$
\left\{\bar{\varepsilon}_{l}\right\}=[B]\left\{\lambda_{0_{i}}\right\},\left\{\bar{\varepsilon}_{n l}\right\}=[A][G]\left\{\lambda_{0_{i}}\right\}
$$

where, $[B]$ and $[G]$ are the product form of the differential operator matrix and the shape functions for the linear and the nonlinear strain vectors, respectively and $[A]$ is the function of displacements associated with the nonlinear strain terms. For the details of $[B],[A]$ and $[G]$ matrices, $[16]$ can be referred.

The strain energy expression can be conceded as:

$$
U_{\varepsilon}^{e}=\frac{1}{2}\left(\left\{\lambda_{0_{i}}\right\}^{T}\left[K_{l}\right]^{e}\left\{\lambda_{0_{i}}\right\}+\left\{\lambda_{0_{i}}\right\}^{T}\left[K_{n l 1}\right]^{e}\left\{\lambda_{0_{i}}\right\}+\left\{\lambda_{0_{i}}\right\}^{T}\left[K_{n l 2}\right]^{e}\left\{\lambda_{0_{i}}\right\}+\left\{\lambda_{0_{i}}\right\}^{T}\left[K_{n l 3}\right]^{e}\left\{\lambda_{0_{i}}\right\}\right)
$$

The expressions for $\left[K_{l}\right]^{e},\left[K_{n l 1}\right]^{e}\left[K_{n l 2}\right]^{e}$ and $\left[K_{n l 3}\right]^{e}$ can be seen in [18].

In the similar manner, the elemental equation of work done due to the in-plane thermal force resultant can be expressed as

$$
W^{e}=\frac{1}{2}\left\{\lambda_{0_{i}}\right\}^{T}\left[K_{G}\right]^{e}\left\{\lambda_{0_{i}}\right\}
$$

where, $\left[K_{G}\right]^{e}=\int_{-1}^{1} \int_{-1}^{1}\left[B_{G}\right]^{T}\left[D_{G}\right]\left[B_{G}\right]|J| d \xi d \eta$ is the elemental geometric stiffness matrix.

The governing equation for the for the post-buckled FG spherical shell panel is obtained by minimizing the total energy expression. This result in

$$
\delta\left(U_{\varepsilon}^{e}-W^{e}\right)=0
$$

Now, substituting Eq. (8) and (9) into Eq. (10), the final form of the equilibrium equation for the post-buckled FG spherical shell panel is conceded to the following form: 


$$
\left(\left(\left[K_{l}\right]+\left[K_{n l 1}\right]+\left[K_{n l 2}\right]+\left[K_{n l 3}\right]\right)-\gamma_{c r}\left[K_{G}\right]\right)\{\Delta\}=0
$$

where, $\gamma_{c r}$ is the critical buckling load factor. $\left[K_{l}\right],\left[K_{n l l}\right],\left[K_{n l 2}\right],\left[K_{n l 3}\right]$ and $\left[K_{G}\right]$ are the corresponding global matrices of $\left[K_{l}\right]^{e},\left[K_{n l l}\right]^{e},\left[K_{n l 2}\right]^{e},\left[K_{n l 3}\right]^{e}$ and $\left[K_{G}\right]^{e}$, respectively.

\section{Results and discussion}

In order to compute the desired responses, a homemade computer code has been developed in MATLAB environment based on the present nonlinear FE model of the FG panel. The critical buckling and post-buckling responses are computed by considering both the TD and TID material properties. For computation, the silicon nitride $\left(\mathrm{Si}_{3} \mathrm{~N}_{4}\right)$ and the stainless steel (SUS304) are considered as the ceramic and the metal constituent at the top and at the bottom surface of the shell panel, respectively. The corresponding TD material properties are taken from the reference and details can be seen in [20].

After establishing the convergence and validation of the present model the effects of different support conditions, power-law index $(n)$ and curvature ratio $(R / a)$ on the post-buckling responses of FG spherical panel under different temperature fields have been investigated. The corresponding responses for FG flat panel are also presented for the sake of comparison. The different support conditions employed for the present analysis can be seen in [16].

\subsection{Convergence and validation studies}

First, a convergence study has been performed to note the exact mesh size at which the present model is capable of computing the linear and nonlinear post-buckling responses for the FG shell panel. The desired responses are computed for $\mathrm{FG}$ spherical $(\mathrm{R} / \mathrm{a}=5, \mathrm{n}=2, \mathrm{a} / \mathrm{h}=10)$ panel with SSSS support condition and flat $(\mathrm{n}=0.2$ and $5, \mathrm{a} / \mathrm{h}=10)$ panel with CSCS support condition and presented in Figure 2 (a) and (b), respectively. The responses are obtained for different mesh sizes and amplitude ratios (Wmax $/ \mathrm{h}=0,0.5,1$ and 1.5 ) by considering various through the thickness temperature distribution (uniform, linear and nonlinear). It is clearly observed that the responses are converging well with mesh refinement for both the spherical and flat panels. Based on the convergence study a $(5 \times 5)$ mesh is used to compute the thermal post-buckling responses throughout the present study.
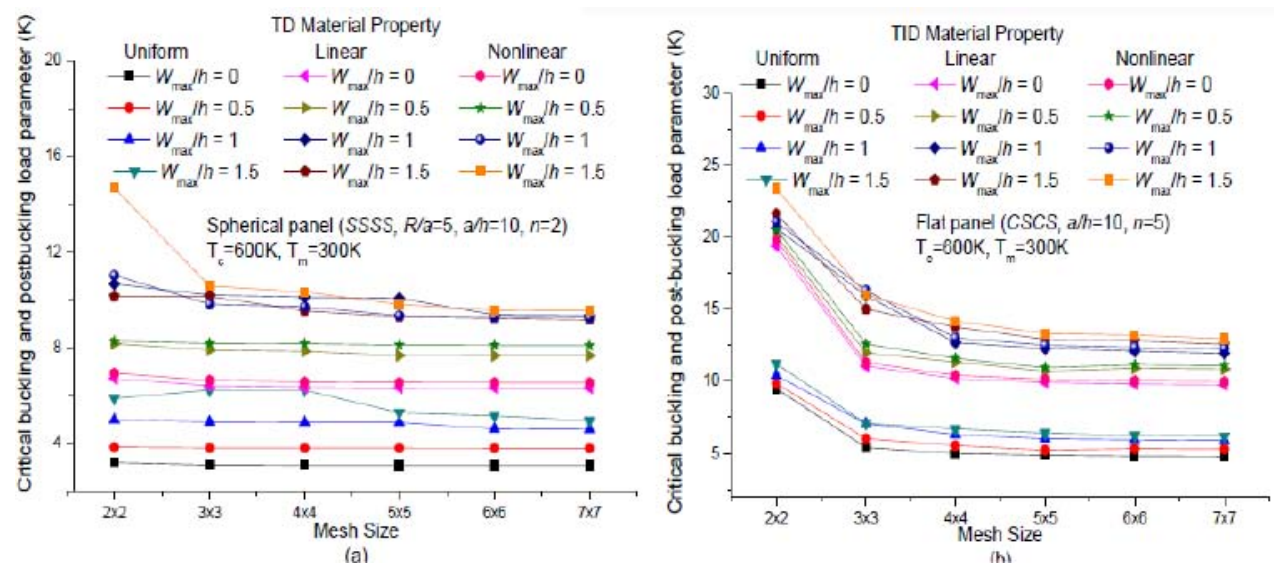

Figure 2. Convergence behavior of the thermal buckling and postbuckling responses of (a) TD-FG spherical panel and (b) TID-FG flat panel. 


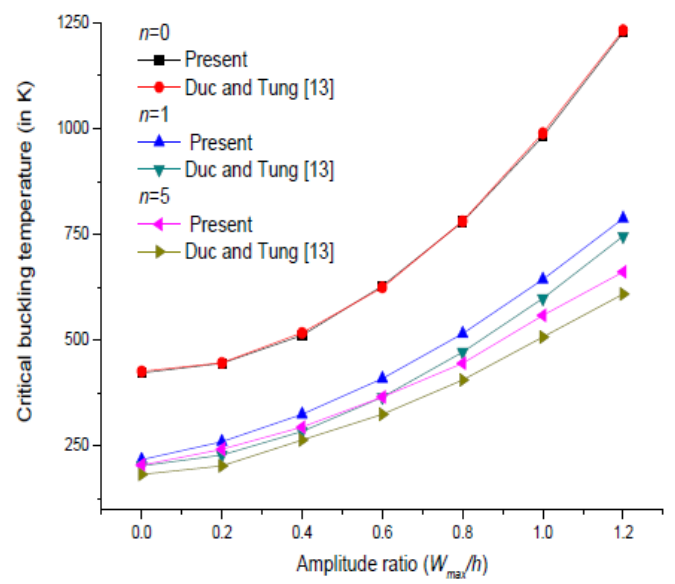

Figure 3. Comparison of the thermal post-buckling responses of simply supported FG (Al/Al2O3) flat panel.

Then the validation of the present model is offered by considering a $\mathrm{FG}\left(\mathrm{Al} / \mathrm{Al}_{2} \mathrm{O}_{3}\right)$ flat $(\mathrm{a} / \mathrm{h}=20$ and $a / b=1)$ panel, as the flat panels are considered to be the simplest form of the shell panel. The thermal post-buckling responses are computed under the uniform temperature rise for three different power-law indices ( $n=0,1$ and 5) and presented in Figure 3 together with the available results of Duc and Tung [13]. The material and geometrical parameters are taken to be the same as in the reference [13]. It is observed that the present results are exhibiting higher critical buckling temperature values as compared to Duc and Tung [13]. It is because, the present model accounts the full geometrical nonlinearity in Green-Lagrange sense whereas, in the reference, the nonlinearity is introduced via von-Karman strain which is unrealistic to consider the exact geometrical distortion under the severe thermal load.

\subsection{Additional Illustrations}

In this section, some more numerical illustrations are presented to show the applicability of the present developed model to compute the post-buckling behavior of FG $\left(S U S 304 / S_{3} N_{4}\right)$ spherical shell panel under varying thermal loading conditions. The temperatures at the bottom and the top of the shell panel are assumed to be $T_{m}=300 \mathrm{~K}$ and $T_{c}=600 \mathrm{~K}$, respectively and both the TD and TID material properties are considered for computation of the responses. To perform a comparative analysis, the responses are also computed for flat panel $(R=\infty)$ under identical geometry, material properties and constraint conditions.

\subsubsection{Influence of temperature field}

The effect of different temperature fields namely uniform, linear and nonlinear temperature distribution through the thickness on the buckling and the post-buckling responses $\left(\gamma_{c r}\right)$ of the square $(a / b=1) \mathrm{FG}\left(S U S 304 / \mathrm{Si}_{3} N_{4}\right)$ spherical $(R / a=10)$ and flat panel $(a / h=10, n=2)$ has been examined in this example. The responses are computed for different support conditions (SSSS and CSCS) and presented in Table 1. It is observed that the critical buckling and post-buckling load parameters are highest for the nonlinear temperature distribution and lowest for all uniform condition. The postbuckling responses are higher for spherical panel than the flat ones. However, the FG spherical panel with the TD properties is showing the inferior buckling and the post-buckling strength in comparison to the FG spherical panel with TID properties. 
Table 1. Effect of support conditions on the critical buckling and post-buckling responses $\left(\gamma_{c r}\right)$ of the FG spherical and flat panel.

\begin{tabular}{|c|c|c|c|c|c|c|c|c|c|c|}
\hline \multirow{3}{*}{$\begin{array}{l}\text { Temperature } \\
\text { field }\end{array}$} & \multirow{3}{*}{$\begin{array}{l}\text { TD/ } \\
\text { TID }\end{array}$} & \multirow{3}{*}{$\begin{array}{c}\text { Support } \\
\text { conditions }\end{array}$} & \multicolumn{4}{|c|}{ Flat Panel } & \multicolumn{4}{|c|}{ Spherical $(R / a=10)$} \\
\hline & & & \multicolumn{4}{|c|}{$W_{\max } / h$} & \multicolumn{4}{|c|}{$W_{\max } / h$} \\
\hline & & & 0 & 0.5 & 1 & 1.5 & 0 & 0.5 & 1 & 1.5 \\
\hline \multirow{4}{*}{ Uniform } & \multirow{2}{*}{ TID } & SSSS & 3.096 & 3.333 & 4.054 & 5.223 & 3.179 & 3.699 & 4.634 & 4.921 \\
\hline & & SCSC & 5.247 & 5.664 & 6.612 & 6.890 & 5.328 & 6.335 & 6.627 & 6.983 \\
\hline & \multirow{2}{*}{ TD } & SS & 2.728 & 2.942 & 3.565 & 3.788 & 2.807 & 3.283 & 4.150 & 4.471 \\
\hline & & & 4.914 & 5.499 & 7.027 & 7.126 & 5.004 & 6.037 & 7.558 & 7.256 \\
\hline \multirow{4}{*}{ Linear } & \multirow{2}{*}{ TID } & & 6.420 & 6.971 & 8.569 & 8.908 & 6.581 & 7.524 & 9.640 & 10.154 \\
\hline & & & 10.903 & 12.148 & 13.493 & 14.373 & 11.051 & 13.172 & 13.703 & 14.792 \\
\hline & \multirow{2}{*}{ TD } & & 5.727 & 6.058 & 7.613 & 8.883 & 5.870 & 6.869 & 8.776 & 9.065 \\
\hline & & & 9.721 & 10.832 & 12.027 & 12.701 & 9.852 & 11.738 & 12.283 & 12.855 \\
\hline \multirow{4}{*}{ Non Linear } & \multirow{2}{*}{ TID } & & 6.628 & 7.175 & 8.749 & 29.375 & 6.793 & 7.948 & 10.130 & 10.431 \\
\hline & & & 11.254 & 12.541 & 13.963 & 14.714 & 11.406 & 13.548 & 14.234 & 15.001 \\
\hline & \multirow{2}{*}{ TD } & & 5.923 & 6.417 & 7.866 & 8.224 & 6.070 & 7.115 & 8.275 & 9.364 \\
\hline & & SCSC & 10.054 & 11.202 & 14.139 & 13.135 & 10.188 & 12.124 & 12.703 & 13.394 \\
\hline
\end{tabular}

\subsubsection{Influence of power-law index}

In this example, the effect of power-law index on the buckling and the post-buckling responses $\left(\gamma_{c r}\right)$ of simply supported thick $(a / h=10)$ square FG $\left(S U S 304 / S i_{3} N_{4}\right)$ spherical $(R / a=5)$ and flat panel with TD and TID properties has been investigated and presented in Figure 4 (a)-(c), respectively. It is noted that, as the FGM becomes metal rich, the post-buckling responses for both spherical and flat panel are decreasing monotonously. This is expected as the ceramic has comparatively higher stiffness than the metal. Also, the FG flat and spherical panel show highest buckling and the post-buckling strength under the nonlinear temperature field in comparison to the other temperature fields.

\subsubsection{Effect of curvature ratio}

It is inferred from the previous examples that the spherical panels exhibit higher buckling and postbuckling strength over the flat panel due to their spatial curvature. Therefore, the buckling and the post-buckling responses $\left(\gamma_{c r}\right)$ of the thin $(a / h=50)$ shallow FG spherical shell panel $(a / b=1, n=2$ and CSCS) under the effect of various curvature ratios $(R / a=10,50,100)$ have been investigated in this example. The post-buckling responses are computed for uniform, linear and nonlinear temperature fields and presented in Figure 5 (a)-(c), respectively. It is observed that the buckling and the postbuckling load parameters are decreasing as the panel tend to become flat.
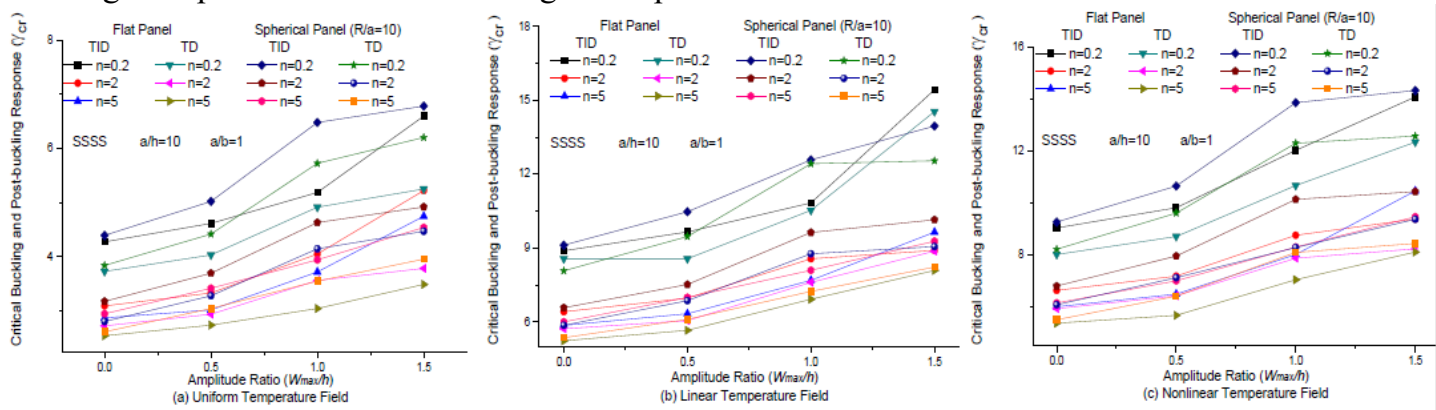

Figure 4. Effect of power-law indices on the critical buckling and post-buckling responses ( $\mathrm{cr} \quad$ ) of FG spherical $(\mathrm{R} / \mathrm{a}=10)$ and flat panel under (a) Uniform (b) Linear and (c) Nonlinear temperature field. 

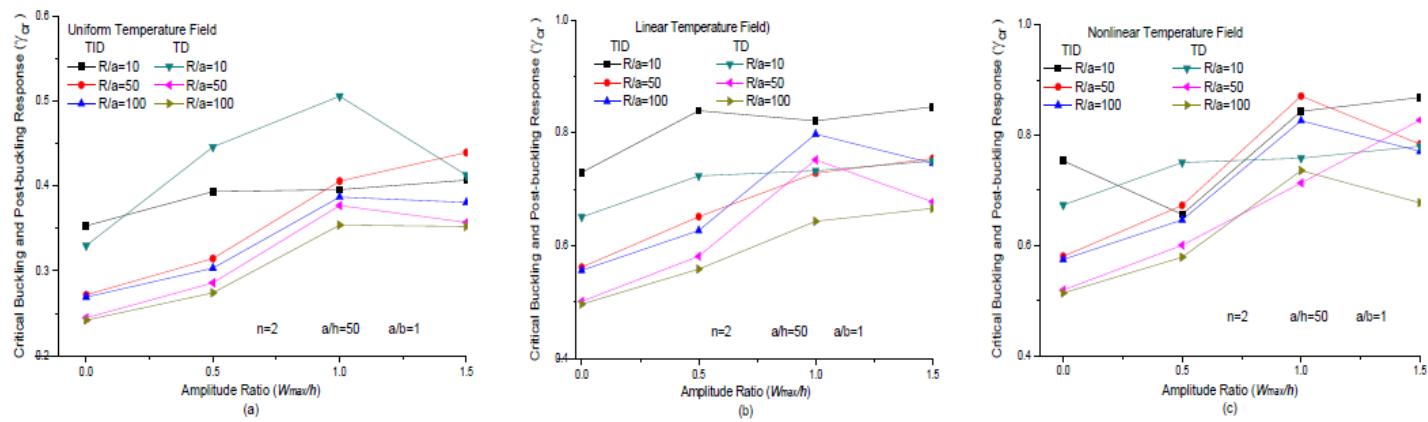

Figure 5. Effect of curvature ratio on the critical buckling and post-buckling responses $\left(\gamma_{c r}\right)$ of FG spherical panel under (a) Uniform (b) Linear and (c) Nonlinear temperature field.

\section{Conclusion}

In this article, the buckling and post-buckling strength of FGM structure have been investigated using a new higher-order model under elevated thermal filed as well as different geometrical configurations. The effective material properties of the FGM are evaluated via the Voigt's micromechanical model in conjunction with the power-law distribution. In addition to this, both the TD and TID material properties of the FG panel are considered in the present analysis. It is observed from the present results that the TD material properties and the Green-Lagrange type geometrical nonlinearity in conjunction with the HOSDT model for the thermal stability of the FG panel structures are inevitable. It is inferred that as the power-law index increase the instability of the FGM panel is enhanced.

\section{References}

1. S. A. M. Ghannadpour, H. R. Ovesy. M. Nassirnia, Comput. Struct. 108(109), 93-99 (2012)

2. H. Huang, Q. Han, N. Feng, X. Fan, Mech. Adv. Mater. Struc. 18(5), 337-46 (2011)

3. X. Zhao, Y. Y. Lee, K. M. Liew, Compos. Struct. 90(2), 161-171 (2009)

4. H. S. Shen, Int. J. Mech. Sci. 49(4), 466-478 (2007)

5. H. Thai, S. Kim, Compos. Struct. 128, 70-86 (2015)

6. K. S. Na, J. H. Kim, Composites Part B 35(5), 429-437 (2004)

7. N. D. Duc, T. Q. Quan, Mech. Compos. Mater. 48(4), 435-448 (2012)

8. H. S. Shen, Int. J. Solids. Struct. 41(7), 1961-1974 (2004)

9. T. L. Wu, K. K. Shukla, J. Huang, Compos. Struct. 81(1), 1-10 (2007)

10. J. S. Park, J. H. Kim, J. Sound Vib. 289(1-2), 77-93 (2006)

11. T. Prakash, M. K. Singha, M. Ganapathi, Engg. Struct. 30(1), 22-32 (2008)

12. K. M. Liew, J. Yang, S. Kitipornchai, J. Appl. Mech. 71(6), 839-850 (2004)

13. N. D. Duc, H. V. Tung, Compos. Struct. 93(11), 2874-2881 (2011)

14. H. S. Shen, H. Wang, Aerosp. Sci. Technol. 38, 9-19 (2014)

15. Kar V R and Panda S K 2015 Composite Structures 129 202-212, (2015)

16. J. N. Reddy, C. D. Chin, J. Therm. Stresses 21, 593-626 (1998)

17. J. N. Reddy, Mechanics of laminated Composite Plates and Shells, Second Edition CRC Press (2004)

18. R. D. Cook, D. S. Malkus, M. E. Plesha, R. J. Witt, Concepts and applications of finite element analysis, Fourth Edition John Willy and Sons (Asia) Pvt. Ltd., Singapore (2009)

19. X. L. Huang, H. S. Shen, Int. J. Solids Struct. 41(9-10), 2403-2427 (2004) 